\title{
La recherche finalisée, une approche ouverte sur la singularité des terrains ${ }^{\star}$
}

\author{
Agathe Dufour ${ }^{1, *}$, Jean-Baptiste Narcy $^{2}$ et Judith Raoul Duval ${ }^{3}$ \\ Q1 ${ }^{1}$ Droit de l'environnement, AScA, Paris, France \\ 2 Sciences de l'environnement, AScA, Paris, France \\ ${ }^{3}$ Économie de l'environnement, Zogma, Val-de-Reuil, France
}

Les différents ministères français en charge de l'environnement de la fin des années 1970 à 2016 ont conduit une expérience de programmation scientifique d'une rare continuité et, avec ses 22 programmes, d'une rare richesse. Trois grands traits la caractérisent: un dualisme fonctionnel des instances chargées du pilotage des programmes (l'association d'un comité d'orientation composé des demandeurs de recherche et d'un conseil scientifique pluridisciplinaire); une ouverture entre les disciplines allant des sciences physiques et biologiques aux sciences de la société en passant par les sciences de l'ingénierie; une ouverture de la recherche sur l'action - et, plus largement même sur les acteurs sociaux. La trace écrite la plus visible du travail effectué dans ce cadre général par les deux organes de direction se trouve dans les appels à proposition de recherche (APR), dans les documents témoignant des activités d'animation (notamment des colloques et séminaires), dans les rapports d'évaluation et dans les publications collectives. L'analyse de ces différents documents assortie d'entretiens avec des personnes ayant joué - ou jouant - un rôle notable dans le fonctionnement des programmes permet de mettre en évidence les façons dont ont été conduites, au niveau de chacun d'eux, les stratégies de recherche destinées à mobiliser les équipes de recherche dans une perspective d'appui aux politiques publiques $^{1}$.

\footnotetext{
‡ Voir dans ce numéro le texte d'introduction de Marcel Jollivet.

*Auteur correspondant : agathe.dufour@asca-net.com

${ }^{1}$ Pour une vue d'ensemble des programmes, $c f$. le texte de Marcel Jollivet, «Une recherche ouverte sur l'action publique» dans ce même numéro de la revue Natures Sciences Sociétés et le rapport d'expertise de l'association Natures Sciences Sociétés-Dialogues «Expertise sur les formes d'ouverture entre disciplines et sur le partenariat en recherche des programmes du MEDDE », 18/11/2014, 63 p. +37 p. (tableaux et figures).
}

Reste à analyser les recherches effectives que ces choix ont produites. Comment l'originalité des orientations de recherche qui résulte de cette finalité se traduitelle en elles? C'est à l'aune du résultat de leur mise en œuvre que les intentions doivent être appréciées. Cet examen comportera deux volets : un portant sur la caractérisation générale de la démarche de recherche, tous programmes confondus, telle que la voient des porteurs de projets et des présidents de conseils scientifiques, un sur la conduite des projets eux-mêmes mettant en rapport leurs objectifs et la façon dont est conçu le processus de recherche visant à les atteindre ${ }^{2}$. La démarche suivie dans cette analyse est rapidement indiquée dans l'encadré ${ }^{3}$.

\footnotetext{
${ }^{2}$ Ce texte est une synthèse de l' ' Étude de caractérisation de la recherche finalisée en appui aux politiques publiques mise en œuvre dans les programmes incitatifs de la recherche de la direction de la recherche et de l'innovation du MEDDE » (39 pages) réalisée par AScA. Le texte intégral de cette étude peut être consulté sur le site d'AScA, http://www.asca-net.com

${ }^{3}$ Il convient de préciser que ce travail n'a pas d'objectif d'évaluation, ni en termes scientifiques, ni en termes de retombées de la recherche. Il n'est pas davantage conçu dans une optique de représentativité. La méthodologie d'échantillonnage employée a consisté à choisir des projets particulièrement illustratifs des démarches que la programmation vise à développer. Il est probable que le résultat de ce choix rende plus compte de ce qui est recherché par le ministère à travers son soutien aux recherches que de la réalité effective de ces recherches considérées dans leur ensemble.
} 


\section{Encadré. La démarche suivie}

Le travail a porté sur les 22 programmes ${ }^{4}$ en cours dans la période 2008-2013. Il a débuté par l'élaboration d'une grille d'analyse fondée sur l'examen de la bibliographie ${ }^{5}$ et sur des entretiens auprès de présidents de conseils scientifiques et de chargés de mission. Cette grille a été utilisée pour un examen d'environ 500 projets, essentiellement sur la base des résumés des recherches. Complétée par des entretiens auprès d'une trentaine de porteurs de projets et par une réflexion de chargés de mission menée en atelier, elle a permis d'identifier cinq familles de projets. Sur cette base, un échantillon de 35 projets a été constitué en vue d'illustrer leur diversité et de mieux repérer les spécificités des différentes familles; ce choix a aussi été fait en veillant à ce que tous les programmes (en fait, 21 sur $22^{4}$ ) soient représentés.

\section{Traits communs caractérisant l'ensemble des recherches soutenues par le ministère}

Les porteurs de projets reconnaissent l'originalité des recherches auxquelles ils participent en répondant aux appels à proposition de recherche (APR) du ministère, que ce soit par rapport à leur propre activité de recherche, et au-delà même, dans le paysage de la recherche pris dans son ensemble. Nos interlocuteurs ont bien conscience que l'horizon de «l'utilité» sociétale des recherches est désormais généralisé : que toute recherche trouve, de près ou de loin, une partie au moins de sa

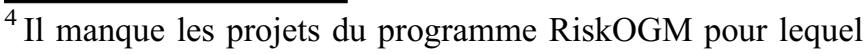
nous n'avons pu disposer d'informations exploitables.

${ }^{5}$ Les ressources bibliographiques mobilisées lors de cette première phase étaient essentiellement les suivantes : journée des présidents de conseil scientifique des programmes de recherche du ministère organisée en janvier 2001 qui avait pour but notamment d'identifier les forces et faiblesses de chacun des programmes. Ce document nous a permis de repérer les éléments sur lesquels porter notre attention en priorité; séminaire «Recherche finalisée et politiques publiques -Contribution à la future loi d'orientation et de programmation de la recherche », septembre 2004 ; l'ouvrage de Christophe Albaladejo, Philippe Geslin, Danièle Magda, Pascal Salembier (Eds.), 2009. La mise à l'épreuve: le transfert des connaissances scientifiques en questions, Versailles, éditions Quae; et dans ce même ouvrage, plus particulièrement, le chapitre de B. Hubert (2009), «La construction en partenariat de recherches sur problèmes: quelle pertinence entre objets et disciplines?» (pp. 209-218).
}

justification dans l'idée que ses résultats auront vocation à éclairer l'action, qu'il s'agisse d'orienter l'action publique ou d'impulser des dynamiques d'innovation. Mais, selon eux, les APR du ministère ont le mérite d'établir une correspondance immédiate, explicite, entre recherche et préoccupations sociétales ou politiques et de le faire autour d'enjeux précis, offrant ainsi aux équipes de recherche l'opportunité rare de s'efforcer d'y répondre par des questionnements scientifiques eux aussi spécifiques. De ce point de vue, la modestie des moyens alloués peut être un atout. Elle permet aux équipes de s'engager sans compromettre leur activité de recherche «classique » et sans prendre le risque de perdre de la visibilité académique. On pourrait parler de recherche «sur mesure» ou «artisanales», par opposition à des activités de recherche que l'on pourrait qualifier d' « industrielles ». On a en quelque sorte affaire à des activités «de niche», au sens où leur volume n'induit pas d'effet structurant sur les stratégies plus générales des équipes répondantes ${ }^{6}$.

Cette appréciation va de pair avec une autre, qui porte sur l'importance qu'en raison de leurs caractéristiques propres (leur finalité d'appui aux politiques publiques et ce qui en découle : l'ouverture entre disciplines et sur les acteurs), les programmes sont amenés à donner une importance particulière aux échanges entre les conseils scientifiques et les équipes, ainsi qu'à l'animation scientifique. Cela se traduit sur deux plans: un interne aux équipes; un entre équipes.

La façon dont les conseils scientifiques assurent leur fonction d'évaluation est décrite comme allant bien au-delà du cadre formel habituel. Prenant souvent la forme d'un suivi particulier, avec le cas échéant désignation d'un référent au sein du conseil scientifique, elle constitue aux yeux des porteurs de projet un investissement, de la part des instances du programme, considéré comme peu habituel dans les enceintes de la recherche. Le dialogue approfondi qui s'instaure ainsi avec les équipes les oblige à une réflexion collective, si ce n'est à des remises en question, inhabituelles. Il amène des chercheurs de disciplines différentes appartenant à la même équipe à sortir de l'isolement disciplinaire dans lequel les cantonne l'évaluation académique, pour nouer des collaborations inédites et susceptibles de se prolonger dans le cadre d'autres projets.

\footnotetext{
${ }^{6}$ En témoigne cette citation: «Grosso modo, si l'on cherche des crédits importants, on se tourne vers les appels d'offres européens; si l'on veut étoffer nos réseaux académiques, on se sert des APR de l'ANR; l'intérêt des APR du ministère est plutôt dans cette possibilité, qui n'existe pas ailleurs, d'aborder des questionnements bien particuliers, attachés à des enjeux sur le terrain, qui nous intéressent».
} 
Quant à l'animation, souci majeur de beaucoup de programmes, elle se situe au niveau du programme et vise à favoriser les échanges entre l'ensemble des équipes qu'il mobilise : outre les publications collectives valorisant les résultats, ce sont surtout les séminaires des programmes, occasions de réelles mises en commun de questionnements, qui sont soulignés comme essentiels. Cette animation transversale très caractéristique des programmes est vue comme une rareté, portée à ce point.

Dans certains cas, nos interlocuteurs ont considéré que ces programmes, outre l'occasion qu'ils donnent de consolider des relations et réflexions entre disciplines, contribuent à l'émergence de véritables communautés scientifiques, qui n'auraient pu se structurer de la sorte ailleurs. En effet, plutôt que sur des dynamiques disciplinaires ou plus largement académiques, ces communautés sont formées sur des objets de politiques publiques (ceux autour desquels sont construits les APR: la biodiversité, les transports, le changement climatique, etc.), voire, dans certains cas, sur des méthodes à expérimenter, adapter ou à développer (modélisation économique, prospective, évaluation, participation de la société civile, etc.) : au terme des dynamiques pluriannuelles des programmes, on peut alors aboutir à de véritables synergies de recherche associant dans la durée, au-delà de la juxtaposition des différents projets, des anthropologues, des économistes, des écologues, des physiciens, des biologistes, etc., se retrouvant régulièrement et acquérant au fil des échanges une culture commune et une «connivence» construite autour de problématiques partagées. Par ailleurs, les chercheurs soulignent que, quand le comité d'orientation joue pleinement son rôle, les APR leur offrent une opportunité rare de fonder ou de consolider des partenariats avec des praticiens de leur domaine; ils disent y trouver des cadres à la fois pratiques et intellectuels pour passer du côtoiement occasionnel à une véritable collaboration avec eux dans une démarche de recherche en partenariat. Il se forme alors de véritables communautés associant scientifiques et porteurs d'action publique.

Toutes ces observations s'incarnent dans un personnage qui est la marque propre de ces programmes : le chargé de mission, tant cheville ouvrière des échanges entre les équipes et les instances que catalyseur d'une communauté en émergence à travers l'animation transversale. D'où l'importance d'assurer une continuité dans l'exercice de ce rôle. Mais d'où aussi la complexité et la délicatesse du rôle, qui incite à penser qu'une formation à le tenir aurait tout son sens.

Un dernier trait commun à l'ensemble des recherches soutenues par le ministère est relatif aux modèles de recherche mobilisés. L'examen de l'ensemble des projets de recherche faisant partie du corpus montre en effet une prégnance très nette du modèle «de terrain », fondé sur l'observation, plutôt que du modèle «du laboratoire» fondé sur le paradigme expérimental ou encore que du modèle de la «recherche-intervention $»^{7}$. Certes, ces deux derniers modèles ne sont pas totalement absents. Il n'en demeure pas moins que le soutien du ministère va en priorité à des projets de recherche fondés sur l'observation de terrain, que celle-ci s'appuie sur les sciences du vivant ou de l'univers, ou sur les sciences humaines et sociales. Ce constat n'est bien sûr guère surprenant, l'environnement ayant émergé en tant qu'objet de recherche au sein de disciplines en général fondées sur ce modèle, telles que, par exemple, l'écologie ou la géographie.

\section{Typologie des projets: les cinq familles}

L'examen de la façon dont le processus de recherche est conçu en fonction des objectifs poursuivis conduit à identifier cinq familles de projets. Ces cinq familles se divisent en deux groupes : trois d'entre elles regroupent les projets qui visent à appuyer la conception et la mise en œuvre des politiques du ministère; les deux autres ont comme objectif de contribuer à susciter l'innovation susceptible de prendre en charge dans le moyen ou long terme les objectifs du ministère.

\section{Appuyer la conception et la mise en œuvre des politiques du ministère}

Les trois familles de projets qui s'y rapportent illustrent trois manières différentes de s'y employer. Les deux premières sont centrées sur la mise en œuvre de ces politiques, la troisième revient sur leur conception même.

Famille 1-Les projets ont comme objectif de fournir des outils ${ }^{8}$, des méthodes ou plus largement des analyses, aux porteurs des politiques pour leur en faciliter la mise

\footnotetext{
${ }^{7}$ Cette distinction reprend celle qui est proposée par A. Hatchuel pour caractériser les rapports entre la recherche et l'action: le modèle de laboratoire et le modèle du terrain sont dans une posture épistémologique qui présuppose que la connaissance précède l'action et que la question posée est celle de son transfert vers celle-ci; le modèle de la rechercheintervention repose sur l'absence de différence fondamentale entre connaissance et action, il implique une association des acteurs au processus de recherche (Hatchuel A., 2000, Intervention research and the production of kwnoledge, in M. Cerf, D. Gibbon, B. Hubert, R. Ison, J. Jiggins, M. Paine, J. Proost, N. Röling (LEARN Group) (Eds.), Cow up a tree. Knowing and learning for change in agriculture. Case studies from industrialised countries. Versailles, Inra Éditions, 55-68). ${ }^{8}$ Par exemple, un outil logiciel produisant des «graphes paysagers » afin de réduire l'impact des infrastructures de transport sur les espèces.
} 
en œuvre. Cette finalité ne doit pas être réduite à sa dimension «instrumentale». Elle peut avoir une portée critique. Alors que les projets sont censés répondre aux difficultés de mise en ouvre d'une politique, deux observations sont à faire : la demande peut émaner autant d'acteurs de la société civile (des associations) que de responsables publics; et, surtout, les chercheurs contribuent par leurs travaux à mieux la formuler, voire même à la formuler tout simplement. Autrement dit, ils jouent un rôle d'accoucheurs; ils co-construisent la demande à laquelle ils sont censés répondre. Ils le font notamment en élargissant la problématique de la recherche, la dégageant ainsi d'une optique trop strictement opérationnelle. En mettant en évidence des points aveugles de la mise en œuvre des politiques publiques, ils incitent à la concevoir dans une perspective non plus seulement planificatrice (quels sont les moyens nécessaires pour atteindre les objectifs fixés?), mais également stratégique (comment créer une dynamique de mise en œuvre susceptible de surmonter les résistances et les inerties ?) ${ }^{9}$.

Par ailleurs, les projets relevant de cette famille ont comme caractéristique de s'adresser à des acteurs intéressés et bien identifiés (services des administrations centrales ou décentralisées) que les chercheurs peuvent associer dès la conception du projet de recherche à leurs travaux, pour en construire ensemble les objectifs et la problématique, échanger des données et en discuter les résultats. Autant d'échanges qui font que les personnes les plus concernées sont en mesure de s'approprier tout le bénéfice de la recherche. Cette osmose se traduit par le recours à des formes particulièrement didactiques et opérationnelles de valorisation («4 pages», "journées techniques » dans le cadre de dispositifs de formation continue à destination de l'administration et/ou «d'acteurs de terrain »...). Il faut souligner que, contrairement à une idée courante, ce souci de coller à l'action n'empêche nullement aux équipes de se consacrer, en parallèle, à la valorisation académique de leurs travaux.

En raison du caractère très finalisé des recherches, les contenus des projets ne procèdent pas d'approches disciplinaires, mais des questions de terrain qui, au contraire, appellent des éclairages émanant de différentes disciplines. Deux caractéristiques croisées des démarches de recherche qui en découlent méritent de retenir l'attention: le montage pluridisciplinaire et la méthode de travail. Lorsque les disciplines concernées relèvent des «sciences et techniques» (par exemple : écologie et informatique pour l'élaboration d'un logiciel; toxicologie, modélisation et évaluation du risque), la démarche de recherche repose sur une décomposition du travail en

\footnotetext{
${ }^{9}$ En mettant en évidence, par exemple les enjeux stratégiques qui se jouent autour des échelles de gouvernance dans la gestion des ressources en eau et ce qui en découle pour la mise en œuvre d'une politique en la matière dans les territoires.
}

« modules » propres à chacune des disciplines, procédant d'un réel partage du questionnement initial, et débouchant ensuite sur une articulation - efficace- des différents résultats de recherche. On retrouve là une culture établie de la recherche finalisée, propre aux sciences de l'ingénieur, associée à des habitudes de travail interdisciplinaire, et dont la légitimité garantit la reconnaissance académique. Lorsque les disciplines relèvent des sciences humaines et sociales (par exemple : sociologie, sciences de gestion, géographie pour la gestion de la ressource en eau), il s'agit davantage d'une mutualisation en continu des méthodes d'investigation (questionnaires d'enquête, bibliographie), des avancées du travail (observations de terrain) et de leur mise en forme (construction et écriture «à plusieurs mains » du rapport scientifique). Le partage des tâches repose moins sur une division disciplinaire du travail que sur une convergence d'éclairages autour de références à des concepts larges (tel que, par exemple, la notion de «coalition d'acteurs») que les différents participants acceptent de mettre en commun.

Famille 2 - Les projets ont l'objectif de contribuer à la mise en œuvre des politiques publiques en étudiant les conditions de leur adaptation aux particularités locales des territoires ${ }^{10}$. Ils interpellent l'acteur public en attirant son attention sur la nécessité de tenir compte de celles-ci dans la mise en œuvre des dispositifs d'action qu'il met en place. Les projets illustrent cette démarche de deux façons: à travers une recherche de terrain visant à donner, à voir et à expliquer les particularités du territoire considéré pour aider à ce qu'elles soient prises en compte $^{11}$; en promouvant, à travers une démarche de recherche-intervention, la mise en œuvre locale d'une politique par la mobilisation d'un potentiel d'expertise ou participatif existant sur place. Là aussi, par leur intervention, les chercheurs aident à la formulation des problèmes et améliorent la façon de les appréhender.

Construire un solide collectif de travail avec le noyau des acteurs locaux les plus concernés (comme prélude à la mobilisation du plus grand nombre pour la valorisation

\footnotetext{
$\overline{{ }^{10} \text { Ces particularités peuvent aussi bien relever des sciences }}$ sociales (lorsqu'elles sont de nature socioéconomique: par exemple, un tissu industriel particulier en vue de la mise en œuvre d'un plan de prévention des risques technologiques) que des sciences du vivant (lorsqu'elles sont de nature écologique : par exemple, la mise en place d'une trame verte et bleue sur la commune de Marseille, etc.).

${ }^{11}$ Par exemple : dans quelle mesure et à quelles conditions des enjeux techniques de gestion du risque inondation en Ile-deFrance peuvent-ils se retrouver dans des territoires de gouvernance en construction, tels que le Grand Paris? Ou : les spécificités écologiques méditerranéennes sont-elles conciliables avec la conception de la Trame Verte et Bleue issue du Grenelle?
} 
des résultats) fait partie de la démarche de recherche: tâche inhabituelle pour les chercheurs, c'est à eux qu'il revient d'établir la confiance entre des acteurs très divers. La sollicitation est stimulante pour les chercheurs en ce qu'elle conduit à mettre à l'épreuve leurs pratiques et leurs cadres de pensée habituels. On retrouve les types d'associations entre disciplines de la famille précédente.

Famille 3 -Les recherches portent non plus sur la mise en œuvre des politiques publiques, mais sur leur conception même, qu'il s'agisse de les adapter ou de mettre en place les fondements de nouvelles. Posant la question de leur pertinence, elles ont une portée politique: observateurs des évolutions de la société et/ ou de l'action publique, les chercheurs jouent, là encore, et plus que dans le cas précédent un rôle d'interpellation : les nouveaux rapports entre sciences et sociétés ne doivent-ils pas conduire à mieux concevoir la participation de la société civile à l'expertise ? La périurbanisation généralisée ne conduit-elle pas s'interroger sur les outils de droit actuels encadrant l'utilisation des sols? Les nouveaux besoins en matière de transports ne doivent-ils pas conduire à revoir les modèles de simulation des modalités de déplacement? Le fait que ces interrogations procèdent de questions posées au sein de la société civile se traduit par la place centrale qui lui est donnée: ou bien les chercheurs en font l'objet de leurs recherches ou bien ils choisissent de l'associer de manière formelle à leur recherche. Dans le premier cas, ils se donnent comme tâche de la représenter en fournissant, à travers leurs analyses, une expression formalisée de ses demandes (on pourrait dire qu'ils se font porteurs de cause). Dans le second cas, ils se situent dans un esprit de rechercheintervention. Dans tous les cas, ce sont les sciences sociales qui sont à la manœuvre. Toutefois, ces interrogations de fond vont, comme il se doit, de pair avec des propositions constructives visant à réorienter les politiques publiques mises en cause. Dans cet esprit, l'équipe de recherche réunit, aux côtés des sciences sociales, les disciplines scientifiques et techniques requises pour traiter les problèmes soulevés. La valorisation des résultats passe soit par les instances politiques nationales (quand la question devient un enjeu politique), soit par la technostructure orchestrant la conception des politiques publiques.

\section{Prolonger l'action du ministère par I'innovation}

Les deux familles qui suivent regroupent des projets qui ne visent plus à aider directement le ministère à mettre en œuvre ses politiques, mais à susciter les innovations susceptibles de lui permettre de servir ses objectifs au-delà de son action immédiate. Cette visée plus générale et à plus long terme s'inscrit dans deux dynamiques, l'une territoriale (famille 4), l'autre sectorielle (famille 5).

Famille 4 -On y retrouve dans les projets la dimension territoriale présente dans la famille 2. Mais l'objet de la recherche n'est pas tel ou tel territoire situé, appréhendé dans ses spécificités propres, ni même le territoire en tant que tel: la question posée est celle, générale, de la territorialisation des politiques publiques (en l'occurrence environnementales) et des conditions de sa réussite. Comment inscrire les finalités et les politiques du ministère dans les dynamiques territoriales, où elles ont vocation à se développer et à produire des effets? Cette question rejoint le courant des sciences politiques qui, depuis une vingtaine d'années, s'intéresse, sur un plan théorique, au mouvement de territorialisation des politiques publiques. Les projets portent notamment sur la manière dont les acteurs du territoire (membres de l'administration déconcentrée ou de services de collectivités locales ou territoriales) se saisissent des finalités ou des dispositifs déployés par le ministère pour leur application locale ${ }^{12}$. Ils s'intéressent en particulier à la «participation», à travers les collectifs «hybrides» associant élus et «société civile», «experts» et «profanes». Ils passent certes par des études de cas localisées fouillées, mais c'est pour aboutir à une formalisation de cadres méthodologiques d'apprentissage collectif destinés aux acteurs territoriaux. Ils analysent en quelque sorte les évolutions des formes de «gouvernance» de l'action publique. Contrairement à ce qu'il en est dans les familles précédentes, ce sont fréquemment les acteurs eux-mêmes qui sont demandeurs. Ce sont donc eux qui formulent, à partir des difficultés auxquelles ils se heurtent dans leurs pratiques, les questionnements initiaux en direction des chercheurs qu'ils sollicitent. Cette "commande» est facilitée par une interconnaissance, voire une collaboration longue, due à une proximité territoriale entre acteurs et équipes de recherche. Portant sur des problèmes locaux, voire conflictuels, elle se transforme volontiers en une recherche-intervention.

L'attrait pour les équipes de recherche est l'opportunité qui leur est offerte d'accéder à des terrains, et d'appuyer méthodologiquement (cela pouvant aller jusqu'à l'expérimentation in situ avec le partenaire) des dynamiques de territorialisation en cours. L'enjeu est commun aux chercheurs et aux acteurs partenaires: il est de se situer à la croisée à la fois des perceptions et des savoirs d'acteurs différents et des dimensions tant physiques et techniques que sociales des questions abordées, en vue d'apprendre collectivement et, ce

\footnotetext{
${ }^{12}$ Par exemple, la rencontre entre une communauté urbaine, soucieuse de prolonger des expérimentations en matière de transport collectif et une équipe de recherche intéressée sur le plan méthodologique par ce genre de question.
} 
faisant, de se construire un référentiel commun ${ }^{13}$. Les croisements à réaliser pour co-construire l'objet luimême et l'action à lui appliquer dans le cadre de la dynamique territoriale passent nécessairement par une association disciplinaire élargie (à l'instar de celle de la famille 2).

Famille 5 - Le souci de promouvoir des innovations susceptibles de produire les changements souhaités par le ministère passe aussi par des filières économiques (agriculture, construction, transports, etc.). L'outil classique dont dispose l'État est l'aiguillon de mesures incitatives. L'objectif des projets de recherche est alors de comprendre comment les acteurs des filières arbitrent entre les contraintes qu'elles leur imposent et les bénéfices qu'ils peuvent escompter en tirer, pour intégrer ou non ces innovations dans leurs activités et stratégies sectorielles. Mais l'inverse peut aussi se produire: une innovation allant dans le sens des objectifs du ministère se heurte à la réglementation en place et à ses évolutions ${ }^{14}$. C'est alors l'innovation qui constitue un aiguillon en incitant à faire évoluer les cadres de l'action publique elle-même. La recherche est alors sollicitée pour expliciter les termes de la contradiction et explorer les voies permettant d'en sortir.

Ces questions conduisent à des pratiques de recherche originales sur deux plans: l'intégration totale ${ }^{15}$ au processus de recherche lui-même des partenaires privés demandeurs (par exemple, des entreprises dotées de services de R\&D) ; la diversité des approches, les projets illustrant les trois modèles évoqués plus haut: le modèle «du laboratoire» (dans le cas d'une recherche en vue d'une innovation technique), peu présent dans les autres familles; le modèle «du terrain» (pour tester par

\footnotetext{
${ }^{13}$ Le paysage (tel qu'il est visé par les politiques publiques, mais aussi tel qu'il est perçu ou souhaité par les habitants d'un territoire), la productivité écologique d'un écosystème côtier (pour la conchyliculture, mais aussi pour l'ensemble des usages et pratiques attachés aux chaînes trophiques en présence); ou, dans un tout autre ordre d'idée, la mobilité (l'offre de transport collectif, mais aussi les formes de «transport personnalisés » tels que le co-voiturage ou le vélopartage) constituent autant d'exemples de ce type de croisements.

${ }^{14} \mathrm{C}$ 'est le cas, par exemple: des conditions d'homologation des véhicules qui limitent les expérimentations sur des camions fonctionnant au gaz (dans le cadre de la lutte contre le changement climatique); des conventions internationales sur la propriété et la brevetabilité du vivant qui bloquent la possibilité de maintenir des pratiques de sélection de semences participatives (pour une agriculture durable), etc. Ces contradictions sont nombreuses.

${ }^{15}$ Les entreprises peuvent être mobilisées pour tester grandeur nature les avancés des projets et transférer aux chercheurs leur savoir-faire.
}

exemple la mise en pratique d'une innovation in situ); le modèle de la recherche-intervention (pour mettre au point une innovation à travers une démarche de co-construction).

Cette diversité des approches se traduit aussi par une diversité des assemblages disciplinaires : le modèle « du laboratoire » donne le pas aux sciences expérimentales; dans le modèle du terrain, ce sont tantôt les sciences du vivant, tantôt les sciences de l'ingénieur, tantôt les sciences humaines qui sont au cœur du projet; dans le modèle de la recherche-intervention, l'ouverture entre disciplines semble plus large, les sciences humaines et sociales introduisant fréquemment apport réflexif, dès la construction du questionnement, aux sciences physiques, biologiques et techniques ${ }^{16}$. On retrouve là une démarche familière aux sciences de gestion. Dans les trois cas de figure, la proximité entre les chercheurs, les experts et les praticiens fait que c'est dans cette famille que les connaissances produites peuvent être vraiment qualifiées d' «hybrides ». Cela va de pair avec une valorisation des résultats de la recherche tournée vers les professionnels et les praticiens, à travers des restitutions professionnelles (synthèses, fiches techniques, d'articles dans des revues professionnelles, voire diffusion par internet) et des publications dans des revues spécialisées. Elle peut aussi déboucher sur une création d'entreprise ou d'un département au sein d'une société existante.

\section{Conclusion}

Procédant d'une même démarche (celle d'un ministère) et ayant un même objectif commun (celui de produire une recherche venant en appui à la mise en œuvre de politiques publiques), les vingt-deux programmes dont il vient d'être question mettaient le ministère face à des enjeux communs de fond et devaient nécessairement être conçus pour y faire face. Le premier de ces enjeux était de se doter d'une démarche de recherche, avec ce que cela implique de précisions dans les opérations de travail à réaliser et de critères de qualité à respecter. Compte tenu de ses objectifs, la façon d'y répondre a consisté à inventer une forme générale de recherche finalisée adaptée à ses besoins et permettant de considérer les programmes comme un ensemble. D'où les traits communs soulignés dans la première partie. D'où, aussi, l'intérêt de les mettre en évidence, car ils constituent l'embryon d'une carte d'identité à construire

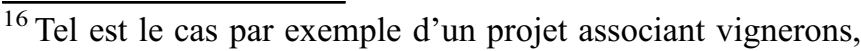
ingénieur agronome et épistémologue pour aboutir à des changements de pratiques viticoles; ou encore d'un autre associant océanographe, historien et praticiens de la pêche pour favoriser la prise en compte de la dimension patrimoniale dans la définition de la durabilité des modes d'exploitation des ressources aquatiques.
} 
de la recherche finalisée, au moins telle qu'elle ressort de l'expérience de programmation ici en cause. Le tableau qui en est proposé s'inscrit dans cette perspective.

Le second enjeu pour le ministère était de pouvoir tirer des recherches les résultats (et même, beaucoup plus largement, les enseignements) précis dont il avait besoin, compte tenu de la multiplicité de ses domaines de compétence, ainsi que de la nouveauté et de la complexité des questions qu'il avait - et qu'il a toujours à traiter. D'où la nécessité que chacun des programmes puisse s'adapter à la diversité des conditions de mise en œuvre de ses politiques. La typologie des familles de projets donne la mesure de la souplesse dont les programmes ont su faire preuve. Et de la richesse de leurs apports sur le plan du savoir-faire tant en matière d'ouverture de la recherche sur elle-même que dans ses rapports à l'action. Cette unité dans la diversité permettant de soumettre les réalités tant naturelles que sociales et politiques à une démarche de recherche à la fois appropriée et évaluable en termes généraux, parce qu'encadrée dans un dispositif aux règles de fonctionnement explicites et adaptées à ses fins, est un legs sans doute précieux et qui mérite attention dans une période où la question des rapports entre la recherche et la société ne cesse de monter en puissance.

\section{Remerciements}

Les auteurs tiennent à adresser leurs vifs remerciements à Marcel Jollivet, pour son intérêt aussi bienveillant qu'exigeant quant au travail exposé ici, et pour son aide précieuse dans la rédaction de cet article.

Citation de l'article : Dufour A., Narcy J.-B., Raoul Duval J., 2017. La recherche finalisée, une approche ouverte sur la singularité des terrains. Nat. Sci. Soc. 25, S29-S35. 